\title{
Evaluación de competencias del docente universitario bajo la percepción de los estudiantes de Ingeniería Civil
}

Evaluation of university teacher's competences according to the perception of Civil Engineering students Avaliação das competências do professor universitário no âmbito da percepção dos estudantes de Engenharia Civil

\author{
REYNALDO MELQUIADES REYES ROQUE ${ }^{1}$
}

\begin{abstract}
RESUMEN
El objetivo general de este trabajo fue determinar la percepción que tienen los estudiantes sobre las competencias del docente universitario, según los factores de planificación, conducción y valoración del proceso de enseñanza aprendizaje. El diseño de investigación es no experimental, transversal y descriptivo; para lo cual se utilizó como instrumento de recolección de información la encuesta; la muestra para el estudio equivale a 250 alumnos del semestre 2019-II de la Escuela Profesional de Ingeniería Civil de la Facultad de Ingeniería Civil de la Universidad Nacional Santiago Antúnez de Mayolo, Huaraz Perú, seleccionados mediante el muestreo aleatorio simple. Los resultados del estudio muestran que los estudiantes en un 54.4\% han percibido las competencias de los docentes a nivel general como bueno y muy bueno, lo que significa una aproximación significativa del proceso de enseñanza aprendizaje de acuerdo a las exigencias para lograr un nivel de calidad de la enseñanza universitaria.
\end{abstract}

Palabras clave: evaluación; competencias; percepción; docente; estudiantes.

\begin{abstract}
The general objective of this work was to determine the students' perception about the university teacher's competences, according to the planning, conduction and assessment factors of the teaching-learning process. The research design is non-experimental, transversal and descriptive; for which the survey was used as an instrument to collect information; the sample for the study is equivalent to 250 students of the 2019-II semester of the Professional School of Civil Engineering of the Faculty of Civil Engineering of the National University Santiago Antúnez de Mayolo, Huaraz-Peru; selected through simple random sampling. The results of the study show that $54.4 \%$ of the students have perceived the competences of the teachers at a general level as good and very good, which means a significant approximation of the process of teaching and learning according to the requirements to achieve a level of quality in university education.
\end{abstract}


Keywords: evaluation; competencies; perception; teacher; students.

\section{RESUMO}

O objetivo geral deste trabalho era determinar a percepção dos estudantes sobre as competências do professor universitário, de acordo com os fatores de planeamento, condução e avaliação do processo de ensino-aprendizagem. O desenho da investigação é não experimental, transversal e descritivo; para o qual o questionário foi utilizado como instrumento de recolha de informação; a amostra para o estudo é equivalente a 250 estudantes do semestre de 2019-II da Escola Profissional de Engenharia Civil da Faculdade de Engenharia Civil da Universidade Nacional Santiago Antúnez de Mayolo, Huaraz-Peru; selecionada mediante amostragem aleatória simples. Os resultados do estudo mostram que 54,4\% dos estudantes consideraram as competências dos professores a nível geral como boas e muito boas, o que significa uma abordagem significativa do processo de ensino e aprendizagem de acordo com os requisitos para alcançar um nível de qualidade no ensino universitário

Palavras-chave: avaliação; competências; percepção; professor; alunos.

\section{INTRODUCCIÓN}

La sociedad actual está inmersa en un proceso de constante cambio que evidentemente, afecta a la educación superior. Tradicionalmente se ha exigido al docente universitario ser un mero contenedor y transmisor de conocimientos, concepción que actualmente está obsoleta. Cada vez se demanda del docente universitario que posea además de saber (competencias científicas), saber hacer (competencias metodológicas/técnicas) saber estar (competencias sociales/participativas) y saber ser (competencias personales) (Sospedra-Baeza et al., 2013). En la evaluación de las competencias del docente universitario bajo la percepción de los estudiantes de Ingeniería Civil, es importante su determinación debido a los cambios y transformaciones que viene sufriendo la educación superior en la actualidad y la calidad de la enseñanza-aprendizaje es un esfuerzo continuo y responsabilidad de cada una de las instituciones que la conforman en el ejercicio de sus funciones misionales centrales: docencia, investigación y proyección social.

Las competencias del profesorado universitario para la atención a la diversidad cambian de acuerdo al tipo de estudiantes que sean abordados, políticas públicas y el contexto geográfico donde desarrollen las prácticas pedagógicas los académicos. Por tanto, las investigaciones exploradas plantean que el profesorado universitario debe poder desarrollar competencias didácticas, pedagógicas y genéricas para desenvolverse en la educación superior. Asimismo, diversos estudios afirman que los docentes tienen que formarse y perfeccionar las competencias pedagógicas, de planificación, metodológicas, de evaluación, investigación y gestión. Estas capacidades se complementan junto con las competencias 
personales y de formación ciudadana. Las competencias transversales son las que contribuyen más a generar transformaciones actitudinales para entender y atender al estudiantado. (Paz-Maldonado, 2018).

En la figura 1 se presentan las competencias básicas y transversales para atender a la diversidad en las instituciones de educación superior.
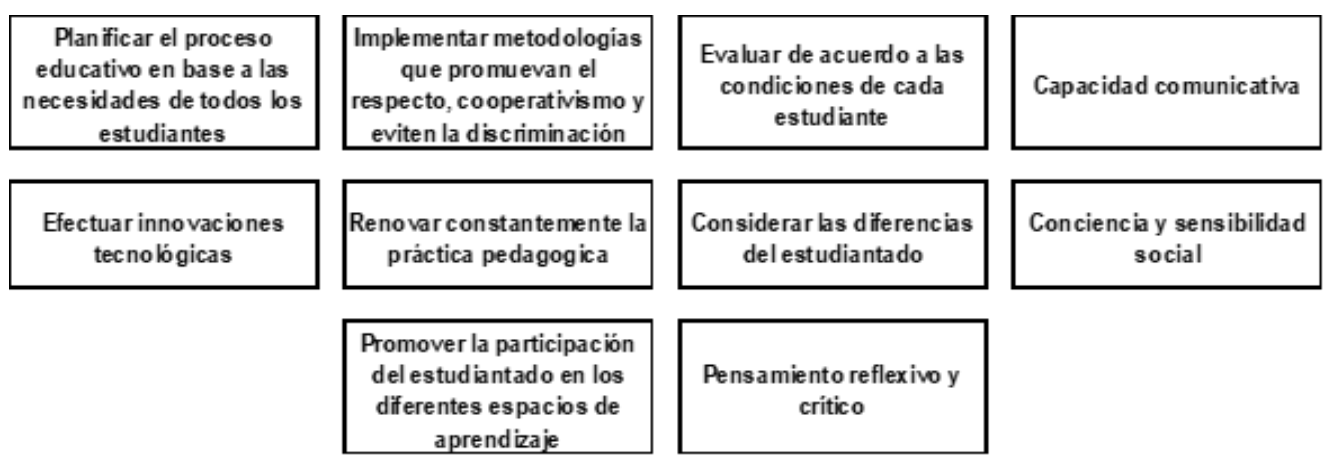

Figura 1. Propuesta sobre las competencias básicas y transversales para atender a la diversidad en la educación superior (Paz-Maldonado, 2018)

Nos encontramos inmersos en la sociedad global del conocimiento donde los cambios sociales, los avances de la tecnología, y la globalización suponen importantes desafíos para la educación universitaria (Rieckmann, 2012). Fernández et al. (2013, p. 865), resaltan que "una de las metas centrales de la Universidad del Siglo XXI es que los estudiantes controlen su proceso de estudio y adquieran competencias que les sean útiles dentro y fuera de las aulas”, pero ¿cómo afrontar el desajuste existente entre lo que los miembros de la sociedad del conocimiento necesitan y lo que los actuales sistemas de Educación Superior están orientados a ofrecer? Gonczi (2001) remarca que el desarrollo de competencias exige una actividad cognitiva compleja donde el sujeto actúe de manera crítica transfiriendo el aprendizaje a diferentes situaciones y ámbitos. Las competencias básicas (generales o transversales) son aquellas competencias transferibles a otros contextos (Bolívar, 2008), relacionadas con el desarrollo personal del individuo, que no dependen de un ámbito temático específico, sino que pueden desarrollarse en distintas disciplinas académicas o profesionales (González \& Wagenaar, 2003; Mir, 2008). En este sentido, entendemos por competencias básicas relacionadas con la evaluación, aquellas competencias transversales que los estudiantes ponen en práctica cuando valoran sus actuaciones y producciones (autoevaluación), las de sus compañeros (evaluación entre iguales) o las del profesorado (heteroevaluación). (Rodríguez et al., 2018). 
Los cambios y transformaciones que ha sufrido la educación superior en la actualidad a nivel mundial, Ospina (2006) plantea que las universidades se enfrentan a desafíos en formar en los estudiantes las competencias necesarias que les permitan desempeñarse en los diversos campos laborales. Este paradigma impone condiciones de exigencias respecto a la competitividad y vigencia de los conocimientos de los profesionales graduados. Por tanto, el docente universitario es uno de los responsables de estimular en los estudiantes los conocimientos propios de la disciplina que enseña y las competencias que son necesarias para la adaptación en el mundo laboral (Daura, 2011). En este sentido, se ha podido constatar en importantes contribuciones de prestigiosos autores e investigadores, la importancia de la evaluación del desempeño del docente universitario para la mejora de la calidad del proceso de enseñanza aprendizaje. La evaluación del desempeño docente en la educación superior se ha realizado por diversos procedimientos y métodos, tales como: la autoevaluación del profesor, la evaluación por pares y responsables académicos y la evaluación por los estudiantes, por solo citar algunos de los procedimientos más difundidos (Tobón et al., 2010; Moreno et al., 2014; Fernandes et al. 2016).

El concepto de competencia se utilizó por primera vez en los años 70, como resultado de investigaciones enfocadas en identificar las variables que permitían explicar el desempeño en el trabajo. Se refieren a un conjunto de conocimientos, habilidades y actitudes que el ser humano aplica para aprender, adaptarse y desempeñarse en el mundo (Camargo \& Pardo, 2008). En el contexto laboral, las competencias constituyen un complejo conjunto de comportamientos que evidencian la capacidad profesional para usar armónicamente sus conocimientos, experiencias, habilidades, disposiciones, actitudes y valores para abordar, resolver o actuar frente a situaciones en el mundo personal, profesional, cívico y social (Camperos, 2007). La competencia corresponde a un saber-hacer en contexto, donde se movilizan recursos cognitivos, integrando conocimientos, habilidades y valores para enfrentarse a distintas situaciones del quehacer en el ámbito profesional (Perrenoud, 2004). En el caso de docentes universitarios, el estudio de las competencias pedagógicas se ha desarrollado internacionalmente con el objetivo de generar perfiles docentes, que permitan guiar el entrenamiento/capacitación y evaluación del profesorado. Enseñar en la universidad actual requiere de conocimientos, habilidades y actitudes diferenciadas de las que se necesitaban 20 años atrás (Villarroel y Bruna, 2017).

Las competencias transversales son aquellas que entregan un valor agregado del proceso de enseñanza y aprendizaje. Funcionan como factor protector del rol del docente, su interacción con los estudiantes y el logro de los aprendizajes esperados. Tiene relación con la capacidad metacognitiva, autocrítica y reflexiva del docente, lo que le permite ponerse en el lugar de otros, vincularse adecuadamente con los alumnos, cuestionar y mejorar sus prácticas docentes. Estas competencias influyen en la efectividad y satisfacción de los estudiantes y docentes con el proceso de enseñanza-aprendizaje. La figura 2, resume este modelo presentado. 


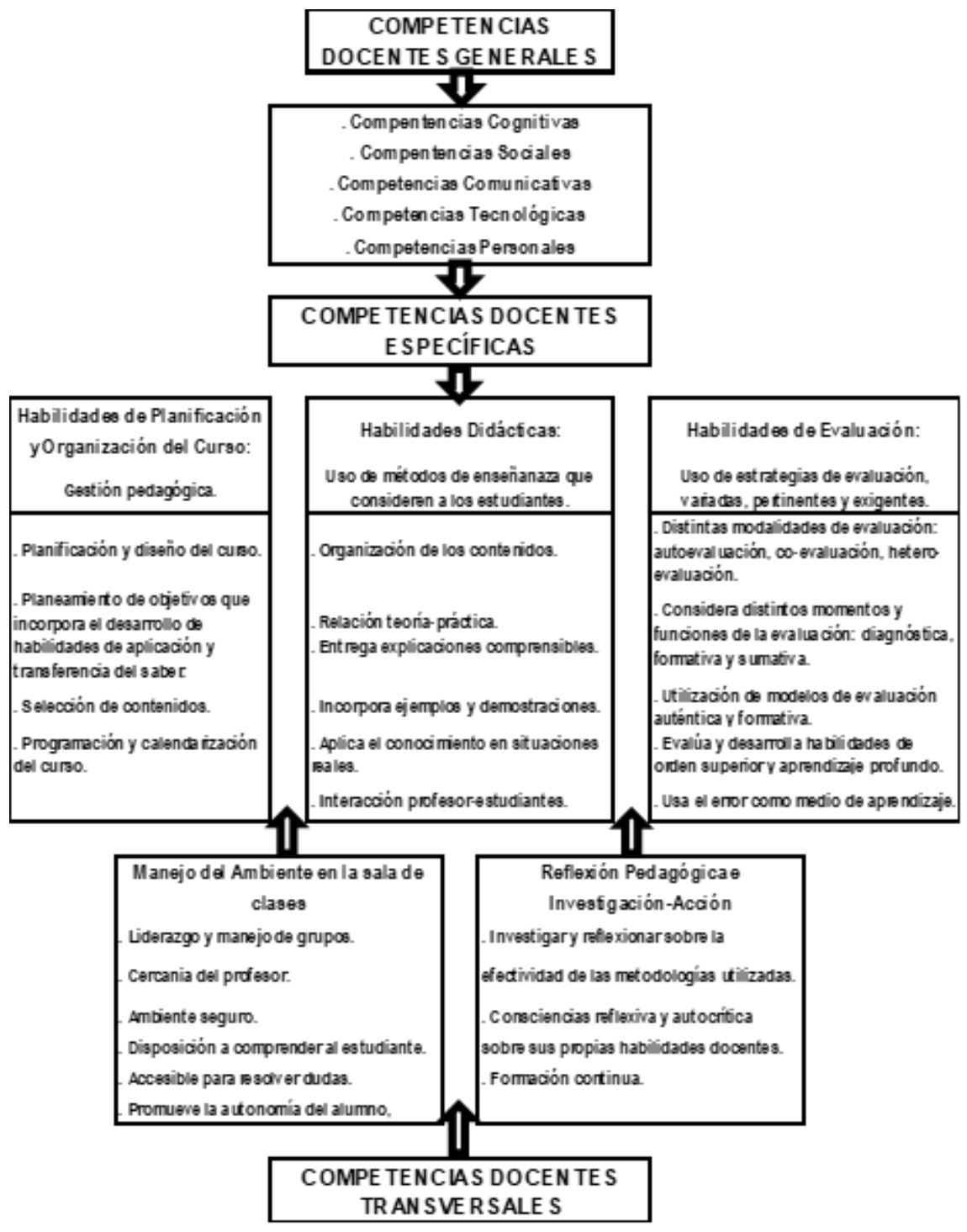

Figura 2. Propuesta de modelo de competencias docentes (Villarroel \& Bruna, 2017)

Según Tomás (2001) "Volver a pensar la Universidad significa reconceptualizar el papel del profesorado, de los estudiantes, de la enseñanza aprendizaje, de la investigación, del gobierno y la gestión", implicando un cambio significativo en la concepción del rol del profesorado universitario, dejando de ser un mero transmisor de información y conocimientos para convertirse en el guía y dinamizador del proceso de enseñanza aprendizaje de los alumnos. En la determinación de las competencias del docente universitario deben participar todos los actores implicados en el acto educativo con el objetivo de planificar correctamente la formación continua de los mismos. Tal y como señalan Flores-Hernández et al. (2011) la evaluación del desempeño docente debe ser considerada una estrategia dirigida a mejorar la educación. La importancia de conocer las demandas del alumno acerca de las competencias que considera necesarias en el profesorado universitario, deviene de la necesidad de aproximar la tarea docente a las inquietudes y necesidades del alumnado, de manera que se optimicé el proceso de E/A, se logre la motivación del alumno facilitando de este 
modo el aprendizaje significativo. Los resultados indican que los estudiantes universitarios de Ingeniería Civil demandan de los docentes, además de las competencias científicas, en mayor medida competencias personales y sociales/participativas. Esto apunta la necesidad de incluir en la programación de la formación continua del profesorado universitario contenidos que potencien competencias personales, sociales y participativas. (Sospedra-Baeza et al., 2013)

Las competencias profesionales son mucho más que una simple acumulación de contenidos (saber), estando también constituidas por habilidades (saber hacer), actitudes y valores (saber ser y estar) (Mas Torelló, 2012). En esta misma línea Echeverría (2002) indica que "para el desempeño eficaz de una profesión es necesario saber los conocimientos requeridos por la misma, un ejercicio eficaz de estos necesita un saber hacer, saber ser y saber estar". En el caso concreto de la docencia universitaria, el saber se corresponde con las competencias científicas, el saber hacer con competencias metodológicas/técnicas, el saber ser con competencias personales y el saber estar con competencias sociales/participativas (figura 3).

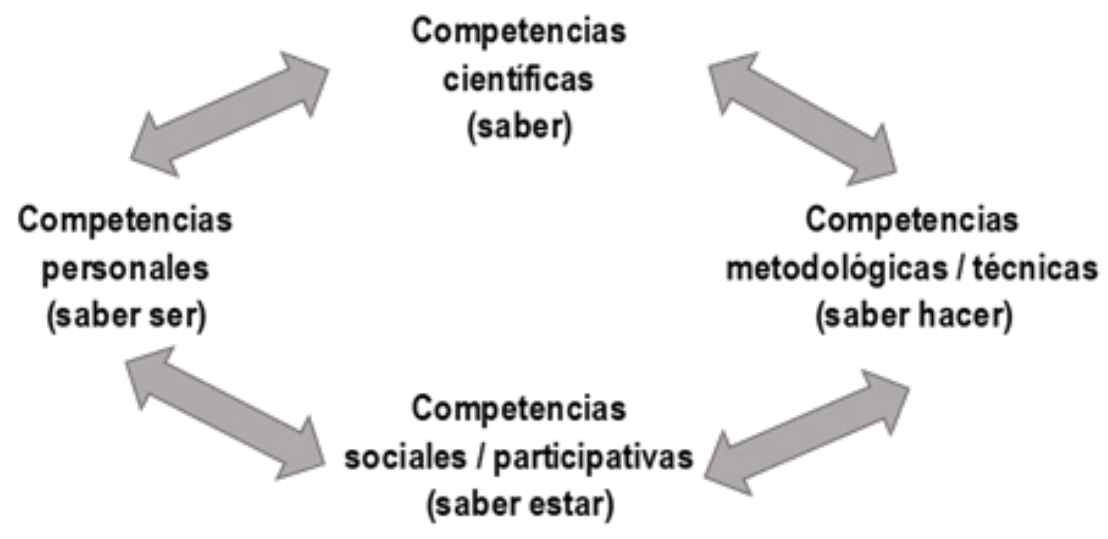

Figura 3. Competencias del profesorado universitario relevantes para el proceso de enseñanza/aprendizaje (Sospedra-Baeza et al., 2013)

La percepción que tienen los alumnos sobre las competencias del docente de la Escuela de Administración de la Universidad Nacional Santiago Antúnez de Mayolo, según los factores de planificación, interacción didáctica del proceso de enseñanza aprendizaje y valoración del proceso de enseñanza aprendizaje. Según un estudio realizado por López et al. (2016), los resultados obtenidos evidencian que la incorporación del enfoque de competencias a la formación universitaria representa una oportunidad para ensalzar el valor formativo de la práctica. Sin embargo, su adopción puede suponer una vuelta a posicionamientos tecnológicos ampliamente cuestionados y una subordinación a las demandas del mercado. Para evitarlo, el panel de expertos propone mejorar las condiciones en las que se ejerce la enseñanza, prestar mayor atención a la formación del profesorado, y ampliar la reflexión y debate en torno al enfoque de competencias y a los sistemas de evaluación (Peláez et al. 2017). 
Consecuentemente, este estudio se justifica porque determina la percepción que tienen los estudiantes sobre las competencias del docente universitario de la Escuela Profesional de Ingeniería Civil de la Facultad de Ingeniería Civil de la Universidad Nacional Santiago Antúnez de Mayolo, Huaraz Perú (EPIC/FIC/UNASAM, 2019-II), según los factores o dimensiones de planificación, conducción (interacción didáctica) y valoración del proceso de enseñanza aprendizaje; habiéndose propuesto como hipótesis general que la percepción que tienen los estudiantes de objeto de estudio sobre las competencias del docente universitario de la Escuela Profesional de Ingeniería Civil es valorada como la de un nivel intermedio o bueno, y en la sección de resultados se aborda la evaluación realizada por los estudiantes que nos darán una aproximación significativa del proceso de enseñanza aprendizaje.

\section{METODOLOGÍA}

El método que se utiliza según el enfoque investigativo es cuantitativo, según su alcance es descriptivo. El tipo de investigación es aplicada porque parte de una situación problemática que requiere ser intervenida y mejorada a favor de la comunidad. Se utiliza el diseño de investigación no experimental, transversal en la modalidad descriptivo. También se utiliza la técnica de revisión bibliográfica, debido a que forma parte integral de toda investigación. La población está compuesta por 430 alumnos de la escuela profesional de Ingeniería Civil, matriculados en el semestre académico 2019-II, de los cuales aleatoriamente se ha extraído una muestra de 250, correspondiendo a 25 alumnos por ciclo. A continuación, se muestra el diagrama investigación:

$$
\mathrm{M} \rightarrow \mathrm{O}
$$

Dónde:

\section{M : Muestra de estudiantes de la EPIC/FIC/UNASAM, 2019-II \\ O : Evaluación de las competencias del docente universitario.}

Luna et al. (2012) documentan el diseño, desarrollo y evidencias iniciales de validez y confiabilidad del Cuestionario de Evaluación de la Competencia Docente con base en la opinión de los estudiantes, así como las teorías implícitas que le subyacen. En este se demuestran las diversas etapas y procesos involucrados, mismos que incluyen la operacionalización del constructo, la validación del contenido mediante juicio de expertos, la aplicación piloto de la versión preliminar, el análisis inicial del comportamiento de los reactivos y del propio cuestionario, la aplicación a una muestra representativa de estudiantes $(\mathrm{n}=400), \mathrm{y}$ la obtención de evidencias de validez y confiabilidad mediante métodos empíricos (análisis de unidimensionalidad, análisis factorial exploratorio e índices de consistencia interna). El trabajo de investigación se realizó en dos etapas: la primera, corresponde al diseño del cuestionario, se enfocó en delimitar el constructo a medir "competencia docente" y en establecer las características del instrumento; en la segunda se realizó el procedimiento cuantitativo para generar evidencias de validez y confiabilidad del instrumento. Finalmente, la estructura del cuestionario después de los análisis realizados y del contraste con el marco teórico quedó tal como se muestra en la Tabla 1, la cual revela los tres factores: planeación y gestión, interacción didáctica en el aula, evaluación y comunicación del proceso enseñan- 
za-aprendizaje, y como indicador tecnologías de la información y la comunicación; y, las sub dimensiones que les corresponden con sus respectivos indicadores y reactivos.

Tabla 1.

Estructura final del Cuestionario de Evaluación de la Competencia Docente

\begin{tabular}{|c|c|c|}
\hline \multirow{13}{*}{$\begin{array}{l}\text { FACTOR } \\
\text { 1.Planeación y } \\
\text { gestión }\end{array}$} & REACTIVO & NO \\
\hline & $\begin{array}{l}\text { Menciona la relación de algunos contenidos del curso con temas de otras } \\
\text { materias del plan de estudios. }\end{array}$ & 1 \\
\hline & $\begin{array}{l}\text { Explica la utilidad de los conocimientos en situaciones de la vida diaria. } \\
\text { Ubica los contenidos del curso en el contexto profesional. }\end{array}$ & 2 \\
\hline & Puntualiza los conceptos importantes de los temas vistos en el curso. & 4 \\
\hline & $\begin{array}{l}\text { Enseña procedimientos para usar el conocimiento en la solución de pro- } \\
\text { blemas reales. }\end{array}$ & 5 \\
\hline & Propone fuentes de información que facilitan el aprendizaje de los temas. & 6 \\
\hline & $\begin{array}{l}\text { En las primeras clases identifica los conocimientos de los estudiantes so- } \\
\text { bre la materia. }\end{array}$ & 7 \\
\hline & $\begin{array}{l}\text { Modifica las actividades o fuentes de información para adecuarlas a las } \\
\text { necesidades del grupo. }\end{array}$ & 8 \\
\hline & $\begin{array}{l}\text { Plantea situaciones con problemas reales que favorecen mi aprendizaje de } \\
\text { los temas. }\end{array}$ & 9 \\
\hline & $\begin{array}{l}\text { Realiza actividades en clase que me motivan a aplicar lo aprendido fuera } \\
\text { del aula. }\end{array}$ & 0 \\
\hline & Estuvo disponible para dar asesorías individuales cuando lo necesite. & \\
\hline & Me motiva a continuar aprendiendo sobre los temas vistos en el curso. & \\
\hline & $\begin{array}{l}\text { Pone en operación actividades en el aula que facilitan el aprendizaje de los } \\
\text { contenidos. }\end{array}$ & 3 \\
\hline \multirow{13}{*}{$\begin{array}{l}\text { 2.Interacción } \\
\text { didáctica en el } \\
\text { aula }\end{array}$} & Aborda los temas con una secuencia razonada. & 4 \\
\hline & El tiempo dedicado para aprender cada tema es suficiente. & 5 \\
\hline & $\begin{array}{l}\text { Acepta sugerencias de los estudiantes sobre la manera de organizar las } \\
\text { actividades. }\end{array}$ & 1 \\
\hline & Da oportunidades iguales a todos los estudiantes para participar en clases. & \\
\hline & Para facilitar la comprensión y conceptos, los define mediante ejemplos. & \\
\hline & $\begin{array}{l}\text { Estructura tareas de aprendizaje que propicien la colaboración entre los } \\
\text { estudiantes. }\end{array}$ & 19 \\
\hline & $\begin{array}{l}\text { Se asegura de que, en las actividades en equipo, cada integrante contribu- } \\
\text { ye a su realización. }\end{array}$ & 20 \\
\hline & $\begin{array}{l}\text { Realiza preguntas a los estudiantes cuando expone, para asegurar la com- } \\
\text { prensión del tema. }\end{array}$ & 21 \\
\hline & Cuando habla, expresa claramente sus ideas. & 22 \\
\hline & $\begin{array}{l}\text { Fomenta la participación del grupo para realizar conclusiones en sus expo- } \\
\text { siciones. }\end{array}$ & 2 \\
\hline & Demuestra ser responsable en su trabajo. & 24 \\
\hline & $\begin{array}{l}\text { Promueve la elaboración de acuerdos de convivencia en el aula con base } \\
\text { en las opiniones de todo el grupo. }\end{array}$ & 2 \\
\hline & Es responsable con todos los estudiantes del grupo. & 2 \\
\hline \multirow{7}{*}{$\begin{array}{l}\text { 3.Evaluación y } \\
\text { comunicación } \\
\text { del proceso } \\
\text { de enseñan- } \\
\text { za-aprendizaje }\end{array}$} & Presenta la lista de temas y su secuencia en el programa de la materia. & \\
\hline & Explica los criterios de evaluación de la materia. & \\
\hline & Manifiesta claramente sus ideas cuando escribe en el pizarrón, & 29 \\
\hline & $\begin{array}{l}\text { Sus exposiciones se caracterizan por tener un inicio, desarrollo y conclu- } \\
\text { sión en sus exposiciones. }\end{array}$ & 30 \\
\hline & $\begin{array}{l}\text { Realiza evaluaciones para retroalimentar sobre las dificultades de aprendi- } \\
\text { zaje. }\end{array}$ & 31 \\
\hline & Respeta los criterios de evaluación planteados al inicio del curso. & 3 \\
\hline & $\begin{array}{l}\text { La evaluación final fue congruente con los contenidos y actividades del } \\
\text { curso. }\end{array}$ & 33 \\
\hline \multirow{2}{*}{$\begin{array}{l}\text { *Tecnologías de } \\
\text { la información y } \\
\text { la comunicación }\end{array}$} & $\begin{array}{l}\text { Utiliza diversos recursos didácticos en clase (por ejemplo, presentaciones } \\
\text { en computadores, videos, fotos, diagramas, etc.) }\end{array}$ & \\
\hline & $\begin{array}{l}\text { Incorpora en sus clases el uso de la tecnología de la información y la co- } \\
\text { municación (por ej. Internet, uso de software, etc.) }\end{array}$ & \\
\hline
\end{tabular}


El cuestionario para evaluar las competencias del docente universitario por los estudiantes, fue validado por Luna et al. (2012) y Peláez et al. (2017). Según la estructura final del cuestionario, representada por los factores planeación, conducción y valoración de proceso de enseñanza aprendizaje, se ha propuesto en la investigación utilizar el cuestionario "percepción de la competencia docente con base en la opinión de los estudiantes".

\begin{tabular}{|c|c|c|c|c|c|c|c|c|}
\hline \multicolumn{9}{|c|}{ Cuestionario: "Percepción de la competencia docente con base en la opinión de los estudiantes" } \\
\hline \multicolumn{9}{|c|}{ 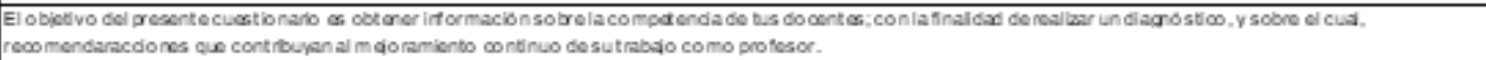 } \\
\hline \multicolumn{9}{|c|}{ 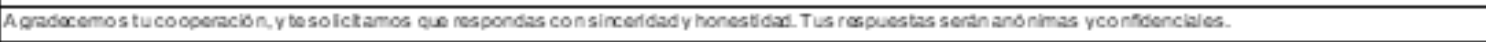 } \\
\hline \multicolumn{9}{|c|}{ 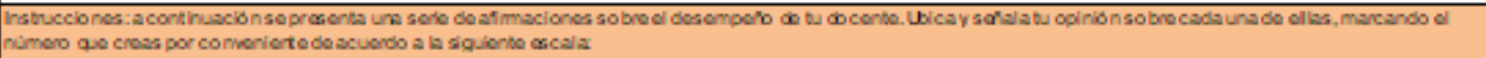 } \\
\hline \multicolumn{3}{|l|}{ Doosnte: } & & & \multirow{2}{*}{$\begin{aligned} \text { Cacl } \\
\text { manos }\end{aligned}$} & \multirow{2}{*}{$\begin{array}{c}\text { Alqunac } \\
\text { vooec }\end{array}$} & \multirow{2}{*}{$\begin{array}{c}\text { Cacl } \\
\text { clompre }\end{array}$} & \multirow{2}{*}{ slempre } \\
\hline \multicolumn{3}{|l|}{ C urco: } & & Numos & & & & \\
\hline Clolo: & \multicolumn{2}{|l|}{$2019-11$} & \multicolumn{2}{|r|}{1} & 2 & 3 & 4 & 6 \\
\hline Dimencilo n & $\begin{array}{c}\text { Sub } \\
\text { dilmencion }\end{array}$ & Indloado $\mathrm{r}$ & $\mathrm{N}^{\circ}$ & \multicolumn{4}{|l|}{ Pregunta } & Opinion \\
\hline \multirow{10}{*}{$\begin{array}{l}\text { P lanif losolon } \\
\text { del proosco do } \\
\text { oncehanzs. } \\
\text { ap rendizaje. }\end{array}$} & \multirow{5}{*}{$\begin{array}{c}\text { Domirio de } \\
\text { los saberes de } \\
\text { la dacipine. }\end{array}$} & 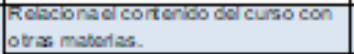 & 1 & \multicolumn{4}{|c|}{ 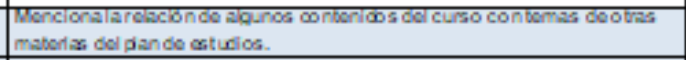 } & \\
\hline & & Ubica los conocim ientos s disdplinares & 2 & \multicolumn{4}{|c|}{ 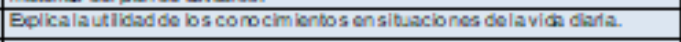 } & \\
\hline & & en cortoceos socides. & 3 & \multicolumn{4}{|c|}{ 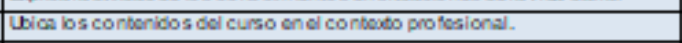 } & \\
\hline & & $\begin{array}{l}\text { A bonda cohnastivamenbolog } \\
\text { conocimientos doclarabvos de cada }\end{array}$ & 4 & $\begin{array}{l}\text { Prociga los concoptos o las } \\
\text { an el curso. }\end{array}$ & Ficiones in & ortantos dol & tomsa vistos & \\
\hline & & $\begin{array}{l}\text { A bonda obhastivamentolos } \\
\text { conocimientos procesimentades de }\end{array}$ & 6 & $\begin{array}{l}\text { Eupocíncalos pasos nocesa } \\
\text { tares. }\end{array}$ & para nesc & run problent & o nadizar una & \\
\hline & & & 8 & Fiesontalaligtadotamánil: & cuondace & pooramad & amaborla. & \\
\hline & & 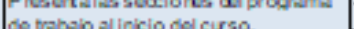 & 7 & 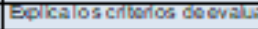 & nos & & & \\
\hline & $\begin{array}{c}\text { Firascion dol } \\
\text { curso dela }\end{array}$ & & 8 & Propone fuertes deinko ms & QQue facti & el aprencizas & dolos bomsa. & \\
\hline & & Plenilca las clases de manom & 8 & moteds & & & & \\
\hline & & 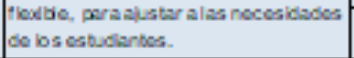 & 10 & $\begin{array}{l}\text { Moditica las sctividsdes o } \\
\text { necesidades del grupe. }\end{array}$ & $\operatorname{res} \alpha$ in & nscion par: & sorusias a las & \\
\hline & & 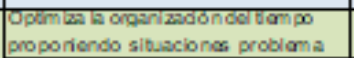 & 11 & 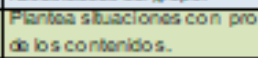 & mas nOSE & 100 tistoroses & mingonowse & \\
\hline & $\begin{array}{l}\text { Gestóndel } \\
\text { progreso de }\end{array}$ & 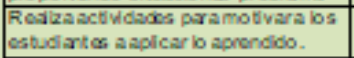 & 12 & 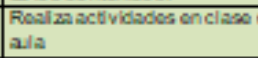 & momotiv & aplicar lo a & onsibo fuen कel & \\
\hline & $\begin{array}{c}106 \\
\text { aprendizipes. }\end{array}$ & 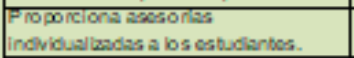 & 13 & Es accesblepander asesor & individual & Eunso lo ne & sito. & \\
\hline & & 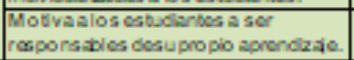 & 14 & Motvaa artuliar holvidusn & $12 \sec$ & comsa vi & inel cursa. & \\
\hline & & 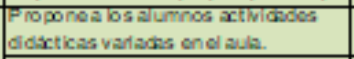 & 15 & 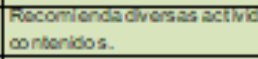 & andast & Sorstisce & nornows/osolos & \\
\hline & & 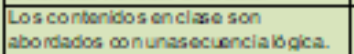 & 18 & A borch los temsa con unas & encia bois & & & \\
\hline & & 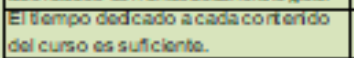 & 17 & 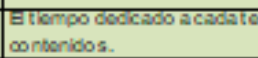 & SSUCE & arad apher & 202000105 & \\
\hline & & 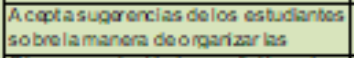 & 18 & $\begin{array}{l}\text { Aced a sugenonclas do bs es } \\
\text { retwidades. }\end{array}$ & Snbos so & Is monarac & porizalas & \\
\hline & $\begin{array}{c}\text { ribersocion } \\
\text { estesticacoel }\end{array}$ & 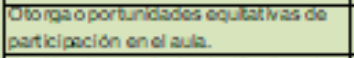 & 10 & Daoportunidases igsies at & 5106050. & ctos para po & Icipar enclase. & \\
\hline $\begin{array}{l}\text { Conduoolon } \\
\text { del pro oe co }\end{array}$ & a.la. & 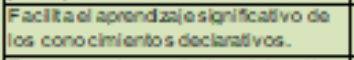 & 20 & Facitala comprensión deco & etos mor & toosientios & oncretos. & \\
\hline $\begin{array}{l}\text { oncehanzs. } \\
\text { ap rendles je. }\end{array}$ & & $\begin{array}{l}\text { Pro mueve ol aproni zalo colabo rativo } \\
\text { en d grupo. }\end{array}$ & 21 & $\begin{array}{l}\text { Eitructura tanoses do apren } \\
\text { aticianbes. }\end{array}$ & Que ph & a la cols & tion entre los & \\
\hline & & $\begin{array}{l}\text { Desarrola la coo persción entre } \\
\text { alumnos. }\end{array}$ & 22 & 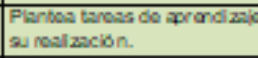 & CQIjpo \&O & cosda intoog & noo contribupea & \\
\hline & & 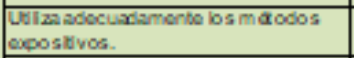 & 23 & $\begin{array}{l}\text { Rediza progintas a los est } \\
\text { compronsiondel toma }\end{array}$ & risc cus & expone, pe & asegrar la & \\
\hline & & Udiza rooursos didsticos diversos. & 24 & 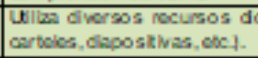 & $\cos$ on & loor aic & Gisoramas, & \\
\hline & & 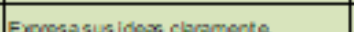 & 25 & Ousno hibla oxposa clarar & Do sua bo & & & \\
\hline & & & 28 & Woritatactaramortesuato & $\infty 10 \mathrm{mn}$ & ERT. & & \\
\hline & & & 27 & Monela una estucbura doinkic & Gesamollo & terreensus & posiciones. & \\
\hline & Comunicación & eseosiciones. & 28 & $\begin{array}{l}\text { Fomenta la participarión de } \\
\text { oposiciones. }\end{array}$ & nopo pora & alwer concl. & fones en sts & \\
\hline & & $\begin{array}{l}\text { Se mantiene acbual zaso en el uso do } \\
\text { latarno lo gia de ia into mocion y }\end{array}$ & 28 & 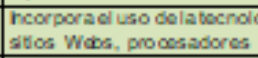 & $\begin{array}{l}\text { 6elainso } \\
\text { otto, ma }\end{array}$ & $\begin{array}{l}\text { Scionylaco } \\
\text { ala mutim }\end{array}$ & 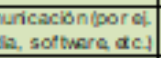 & \\
\hline & & $\begin{array}{l}\text { Ejerco sutrabejo consertibo do } \\
\text { raponamillad. }\end{array}$ & 30 & Demuartiaser nesponsabie & sutrabajo. & & & \\
\hline & Valones & 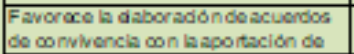 & 31 & 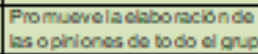 & ondos doc & wivencla on & sisaconbagen & \\
\hline & & Acbiaconnespebo hacla las personas & 32 & Es nespetisso con $60006 \mathrm{bs}$ & dojiates & onpo. & & \\
\hline Valo raolo n del & Evaluscion del & $\begin{array}{l}\text { Rediza evaluscio nes con fincs } \\
\text { formabvos duranto ol curso. }\end{array}$ & 38 & 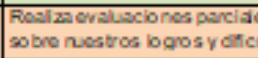 & $\begin{array}{l}\text { b lagoo } \\
\text { los. }\end{array}$ & rso parare & Iimontamos & \\
\hline $\begin{array}{l}\text { proosco do } \\
\text { oncohanza. }\end{array}$ & proceso & Redizaevaluscionsumatvaal fnal od & 34 & Respetalos crtentos de ovel & ion plantio: & s al inicio oc & curso. & \\
\hline & & & 35 & La ereluadón final tue acono & onlos $\infty r$ & ios y activi: & vides del arso. & \\
\hline
\end{tabular}

Figura 4. Estructura final del Cuestionario de Evaluación de la Competencia Docente 
Para el análisis estadístico, se ha se utilizado el programa estadístico SPSS, inicialmente los resultados del cuestionario han sido agrupados en una hoja Excel, para luego exportar al programa y realizar el análisis estadístico y su interpretación. Los resultados serán mostrados haciendo uso de tablas y gráficos.

\section{RESULTADOS}

De acuerdo a la recolección de datos a partir de las encuestas realizadas, nos generó información importante que responden directamente al objetivo de la investigación, en ese sentido se muestran los siguientes resultados:

- Resultados de la evaluación de las competencias del docente universitario bajo la percepción de los estudiantes de la EPIC/FIC/UNASAM, 2019-II en general: Planificación + Conducción + Valoración del proceso enseñanza aprendizaje.

Tabla 2.

Percepción de los estudiantes de la EPIC/FIC/UNASAM, 2019-II, sobre las competencias del docente en general (Planificación + Conducción + Valoración del proceso enseñanza aprendizaje)

\begin{tabular}{lll}
\hline Grado & $\mathbf{N}$ & $\mathbf{\%}$ \\
Malo & 24 & 9.6 \\
Regular & 90 & 36.0 \\
Bueno & 107 & 42.8 \\
Muy Bueno & 29 & 11.6 \\
Total & 250 & 100.0 \\
\hline
\end{tabular}

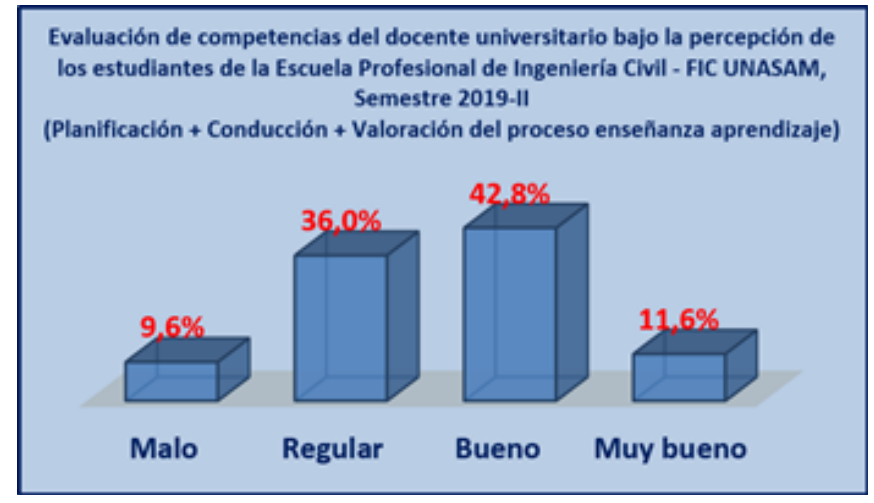

Figura 5. Evaluación de las competencias del docente universitario bajo la percepción de los estudiantes de la EPIC/FIC/UNASAM, 2019-II, en general: Planificación + Conducción + Valoración del proceso enseñanza aprendizaje.

Los resultados indican que el 9.6\% de los estudiantes perciben las competencias docentes como malo, el $36.0 \%$ como regular, el $42.8 \%$ como bueno y el $11.6 \%$ como muy bueno respectivamente. 
- Resultados de la evaluación de las competencias del docente universitario bajo la percepción de los estudiantes de la EPIC/FIC/UNASAM, 2019-II, sobre planificación, conducción y valoración del proceso enseñanza aprendizaje.

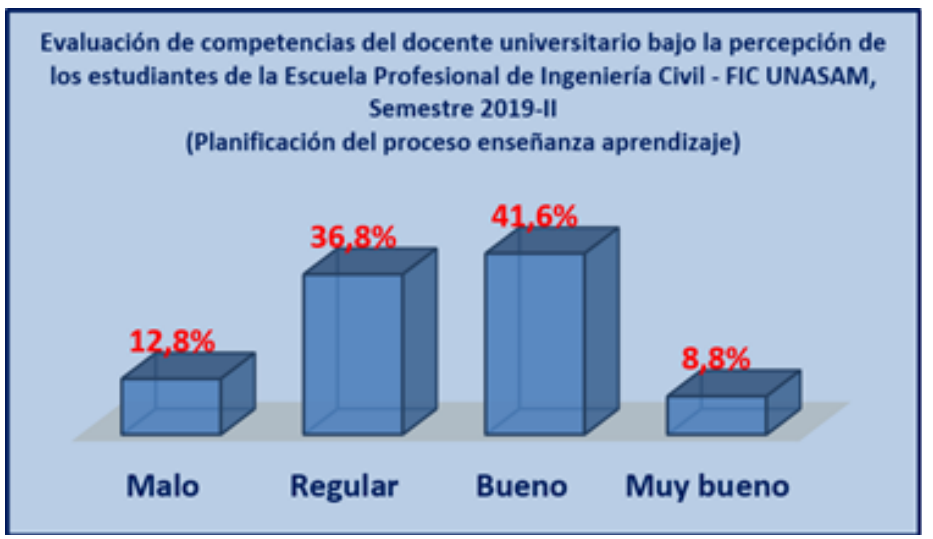

Figura 5. Evaluación de las competencias del docente universitario bajo la percepción de los estudiantes de la EPIC/FIC/UNASAM, 2019-II: Planificación del proceso enseñanza aprendizaje

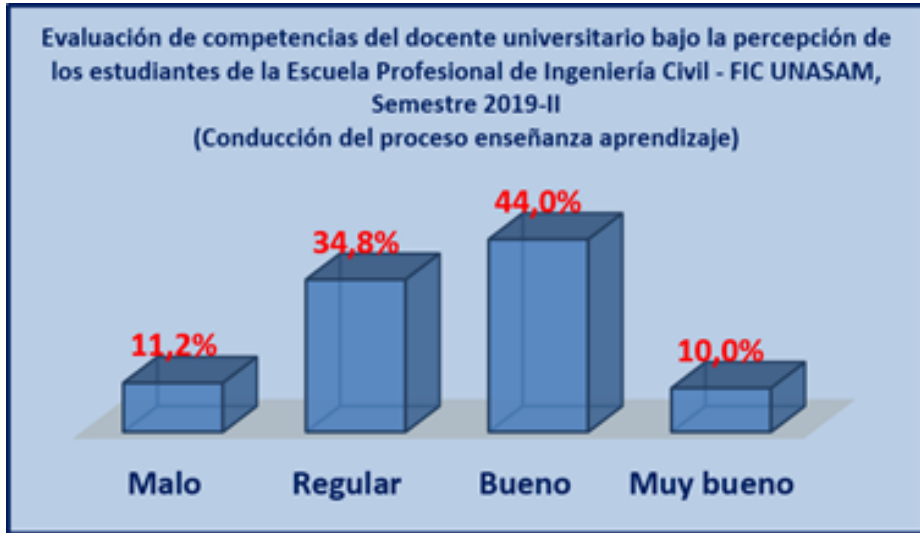

Figura 6. Evaluación de las competencias del docente universitario bajo la percepción de los estudiantes de la EPIC/FIC/UNASAM, 2019-II: Conducción del proceso enseñanza aprendizaje

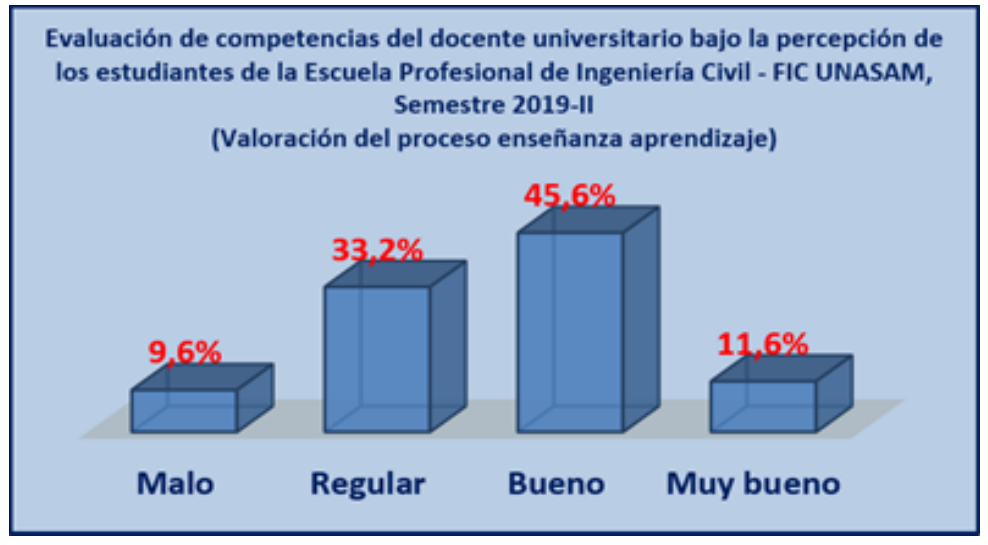

Figura 7. Evaluación de las competencias del docente universitario bajo la percepción de los estudiantes de la EPIC/FIC/UNASAM, 2019-II: Valoración del proceso enseñanza aprendizaje 
En las figuras 5, 6 y 7, se muestran los resultados de los porcentajes de percepción de los estudiantes sobre las competencias de los docentes universitarios como malo, regular, bueno y muy bueno; para las dimensiones de planificación, conducción y valoración del proceso enseñanza aprendizaje respectivamente.

- Resultados de la contrastación de hipótesis

Tabla 3.

Contrastación de hipótesis usando la tabla de contingencia y la prueba chi cuadrado

Tabla de contingencia - Percepción de las competencias

\begin{tabular}{|c|c|c|c|c|}
\hline Encuestas a estudiantes & Malo & Regular & Bueno & Muy bueno \\
\hline Estudiantes $\leq 5^{\circ}$ ciclo & 7.48 & 40.12 & 47.82 & 9.87 \\
\hline Estudiantes $6^{\circ}$ al $10^{\circ}$ ciclo & 8.18 & 39.54 & 48.65 & 10.14 \\
\hline \multicolumn{5}{|c|}{ Ji cuadrada $=0.9056$} \\
\hline
\end{tabular}

\section{DISCUSIÓN}

Con los resultados de la investigación, se ha realizado el análisis respectivo sobre la evaluación de las competencias del docente universitario bajo la percepción de los estudiantes de la EPIC/FIC/UNASAM, 2019-II, como sigue:

Los resultados de acuerdo a la tabla 3, muestran que los estudiantes durante el semestre 2019-II han percibido las competencias de los docentes a nivel general como bueno y muy bueno, situación explicada por el hecho de que el 54.4\% $(42.8+11.6 \%)$ de los estudiantes percibe las competencias por encima de bueno; y se tiene que un $45.6 \%(9.6 \%+36.0 \%)$ de los estudiantes perciben las competencias como malo y regular, porcentaje este último bastante alto, que estaría indicando que aún falta mejorar las competencias de los docentes universitarios de la EPIC/FIC/UNASAM, 2019-II, en las dimensiones de planificación, conducción y valoración del proceso enseñanza aprendizaje respectivamente, lo cual tienen que ver con la capacidad para dominar un área determinada, aprender a tratar con tareas bien definidas y brindar respuestas efectivas para la resolución de problemas en un ámbito profesional concreto, con el fin de lograr un nivel de calidad de la enseñanza universitaria significativo. Estos resultados se contrastan con lo indicado por Rodríguez et al. (2018), concluyendo que de acuerdo a los principales resultados obtenidos, en primer lugar se destaca la necesidad de diseñar espacios donde los estudiantes universitarios reciban oportunidades y apoyo en el desarrollo y adquisición de las destrezas que necesitan para llegar a ser aprendices independientes y autónomos, ya que las competencias percibidas con menor grado de desarrollo por los estudiantes son aquellas relacionadas con la capacidad reflexiva y la autonomía del individuo, competencias necesarias para poder desenvolverse en un contexto profesional y social complejo y cambiante.

En las figuras 5, 6 y 7 aparecen los resultados de la evaluación por los factores o dimensiones de planificación, conducción y valoración del proceso de enseñanza aprendizaje, los cuales se correlacionan bien con los resultados obtenidos en la tabla 3 y la figura 4 que evalúa en forma en general los procesos de enseñanza aprendizaje involucrados (Planificación 
+ Conducción + Valoración). Estos resultados guardan relación con Sospedra-Baeza et al. (2013) que indican que los estudiantes de la EPIC/FIC/UNASAM, 2019-II valoran como más importantes las competencias científicas y, entre estás la más valorada es el conocer ampliamente los contenidos científicos de la materia que se imparte, competencia que tradicionalmente se ha identificado como la más importante del docente universitario, es decir, desde la concepción clásica del profesor como mero transmisor de conocimientos.

De acuerdo a los resultados obtenidos de la contrastación de hipótesis, con relación a la prueba de contingencia realizada agrupando la valoración de los alumnos que cursan entre el $1^{\circ}$ ciclo al $5^{\circ}$ ciclo y los alumnos que cursan entre el $6^{\circ}$ ciclo al $10^{\circ}$ ciclo; se observa que la prueba Chi cuadrada muestra un valor de 0.91 para un valor teórico de 3.59 , indicando diferencias en las percepciones que tienen los estudiantes de ambos grupos analizados en la EPIC/FIC/UNASAM, 2019-II. Estos resultados son concordantes con la investigación realizada por Fernandes et al. (2016) quienes concluyen que el análisis de las percepciones de estudiantes y docentes sobre el instrumento de evaluación del desempeño docente en una universidad pública que deben emplearse para esta evaluación son: las competencias pedagógico-didácticas, investigativas, de extensión universitaria y de gestión académica. De acuerdo con las percepciones de estudiantes y docentes, la competencia que priorizan en la evaluación del desempeño docente es la competencia pedagógico-didáctica, en otras palabras, que el profesor facilite el aprendizaje. Sin embargo, no hay coincidencia de criterios en cuanto al orden de importancia que le confieren a las demás dimensiones. Los estudiantes consideran que deben valorarse las competencias de extensión universitaria, de gestión académica e investigativas en este orden de prioridad; mientras los docentes priorizan las competencias de gestión académica, las investigativas y de extensión universitaria.

\section{CONCLUSIONES}

Respecto al objetivo general del estudio se ha determinado que las competencias de los docentes a nivel general como bueno y muy bueno, situación explicada por el hecho de que el $54.4 \%$ de los estudiantes percibe las competencias por encima de bueno; y se tiene que un $45.6 \%$ de los estudiantes perciben las competencias como malo y regular, porcentaje este último bastante alto; concluyéndose que aún falta mejorar las competencias de los docentes universitarios de la EPIC/FIC/UNASAM, 2019-II, en las dimensiones de planificación, conducción y valoración del proceso enseñanza aprendizaje respectivamente, lo cual tienen que ver con la capacidad para dominar un área determinada, aprender a tratar con tareas bien definidas y brindar respuestas efectivas para la resolución de problemas en un ámbito profesional concreto, con el fin de lograr un nivel de calidad de la enseñanza universitaria significativo.

De los resultados de la evaluación por los factores o dimensiones de planificación, conducción y valoración del proceso de enseñanza aprendizaje, se concluye que estos se correlacionan bien con los resultados obtenidos de la evaluación en forma en general los procesos de enseñanza aprendizaje involucrados (Planificación + Conducción + Valoración), además estos resultados indican que los estudiantes de la EPIC/FIC/UNASAM, 2019-II, valoran como más importantes las competencias científicas y, entre estás la más valorada es el conocer ampliamente los contenidos científicos de la materia que se imparte, competencia 
que tradicionalmente se ha identificado como la más importante del docente universitario, es decir, desde la concepción clásica del profesor como mero transmisor de conocimientos.

\section{REFERENCIAS}

Bolívar, A. (2008). El discurso de las competencias en España: educación básica y educación superior. Revista Electrónica de Docencia Universitaria, 6(2). https://revistas. um.es/redu/article/download/35241/33761

Camargo, I., \& Pardo, C. (2008). Competencias docentes de profesores de pregrado: Diseño y validación de un instrumento de evaluación. Universitas Psychologica, 7(2), 401- 455 .

Camperos, M. (2007). La evaluación por competencias, mitos, peligros y desafíos. Educere, 43, 805- 814 .

Daura, F. (2011). Las estrategias docentes al servicio del desarrollo del aprendizaje autorregulado. Estudios Pedagógicos, 37(2), 77-88. http://www.scielo.cl/pdf/ested/v37n2/ art04.pdf

Echeverría. B. (2002). Gestión de la competencia de acción profesional. Revista de Investigación Educativa, 20(1), 7-43.

Fernandes, D., Sotolongo, M., \& Martínez, C. (2016). La Evaluación del Desempeño por Competencias: Percepciones de Docentes y Estudiantes en la Educación Superior. Formación Universitaria, 9(5), 15-24. https://scielo.conicyt.cl/scielo.php?script=sci_abstract\&pid=S0718-50062016000500003\&lng=es\&nrm=iso

Fernández, E., Bernardo, A., Suárez, N., Cerezo, R., Núñez, J., \& Rosário, P. (2013). Predicción del uso de estrategias de autorregulación en la educación superior: Un análisis a nivel individual y de contexto. Anales de Psicología, 29(3), 865-875. http:// dx.doi.org/10.6018/analesps.29.3.139341

Flores-Hernández, F., Martínez-González, A., Sánchez-Mendiola, M., García-Cabrero, B., \& Reidl, L. (2011). Modelo de competencia docente del profesor de medicina en la UNAM. RELIEVE, 17(2), 11-21.

Gonczi, A. (2001). Análisis de las tendencias internacionales y de los avances en educación y capacitación laboral basadas en normas de competencias. Educación y capacitación basadas en normas de competencias. Limusa.

González, J., \& Wagenaar, R. (2003). Tuning Educational Structures in Europe. Final Report Phase One. Universidad de Deusto.

López, C., Benedito, V., \& León, M. (2016). El enfoque de competencias en la formación universitaria y su impacto en la evaluación: la perspectiva de un grupo de profesionales expertos en Pedagogía. Formación universitaria, 9(4), 11-22. Formación universitaria, 9(4), 11-22. https://scielo.conicyt.cl/scielo.php?pid=S0718-50062016 000400003\&script $=$ sci_arttext

Luna, E., Calderón, N., Caso, J., \& Cordero, G. (2012). Diseño, desarrollo y validación de un cuestionario de evaluación de la competencia docente por los estudiantes. $R E$ VALUE: Revista de evaluación educativa, 1(1). 1-22. https://www.researchgate.net/ publication $/ 277874710$ 
Mas Torelló, O. (2012). Las competencias del docente universitario: la percepción del alumno, de los expertos y del propio protagonista. REDU - Revista de Docencia Universitaria, 10(2), pp. 299-318.

Mir, A. (2008). Las competencias transversales en la Universidad Pompeu Fabra. La visión de los docentes y estudiantes de segundo ciclo. REDU: Revista de Docencia Universitaria, Número monográfico dedicado a Formación centrada en competencias, 1(6), 1-16. https://revistas.um.es/redu/article/download/10641/10231

Moreno, M. P., Nava, M. C., \& Campos, M. (2014). Los comentarios abiertos como referente de evaluación de la docencia universitaria: La conveniencia de su interpretación y tratamiento. Formación universitaria, 7(1), 41-48. https://scielo.conicyt.cl/scielo. php?pid=S0718-50062014000100006\&script $=$ sci_arttext\&tlng=en

Ospina, A. (2006). Currículo por competencias en la Universidad de La Sabana. Rev. Aquichan, 6(1), 117-124. http://www.scielo.org.co/pdf/aqui/v6n1/v6n1a11.pdf.

Paz-Maldonado, E. (2018). Competencias del Profesorado Universitario para la Atención a la Diversidad en la Educación Superior. Revista Latinoamericana de Educación Inclusiva, 2018, 12(2), 115-131. https://www.researchgate.net/publication/328923635

Peláez, G., Vílchez, J., Valderrama, L., \& Carbajulca, M. (2017). Evaluación de las competencias del docente bajo la percepción de los estudiantes de la escuela profesional de administración de la Universidad Nacional Santiago Antúnez de Mayolo, 2016. Aporte Santiaguino, 10(1), 119-130. https://doi.org/10.32911/as.2017.v10.n1.188

Perrenoud, P. (2004). Diez nuevas competencias para enseñar. Graó.

Rieckmann, M. (2012). Future-oriented higher education: Which key competencies should be fostered through university teaching and learning? Futures, 44(2), 127 135. http://ahero.uwc.ac.za/index.php/?module=cshe\&action=downloadfile\&file$\mathrm{id}=18409092513184580308257$

Rodríguez, G., Ibarra, M., \& Cubero, J. (2018). Competencias básicas relacionadas con la evaluación. Un estudio sobre la percepción de los estudiantes universitarios. Educación $X X 1,21(1), 181-207$. https://www.redalyc.org/pdf/706/70653466009.pdf

Sospedra-Baeza, M. J., Lloret-Catalá, M. C., \& Cañas-Louzau, T. R. (2013). Percepción de los estudiantes de ingeniería civil sobre las competencias óptimas del docente universitario. Sinéctica, (41), 2-20. http://www.scielo.org.mx/scielo.php?pid=S1665-1 09X2013000200012\&script=sci_arttext

Tobón, S., Pimienta, J. H., \& García, J. A. (2010). Secuencias didácticas: aprendizaje y evaluación de competencias. Pearson Educación de México, S.A. de C.V. http:// files.ctezona141.webnode.mx/200000004-8ed038fca3/secuencias-didacticastobon-120521222400-phpapp02.pdf

Tomás, M. (2001). Presentación. Educar, 28, 6-9.

Villarroel, V., \& Bruna, D. (2017). Competencias Pedagógicas que Caracterizan a un Docente Universitario de Excelencia: Un Estudio de Caso que Incorpora la Perspectiva de Docentes y Estudiantes. Formación Universitaria, 10(4), 75-96. https://scielo. conicyt.cl/pdf/formuniv/v10n4/art08.pdf 